Www.jmscr.igmpublication.org

Impact Factor (SJIF): 6.379

Index Copernicus Value: 79.54

ISSN (e)-2347-176x ISSN (p) 2455-0450

crossrefDOI: https://dx.doi.org/10.18535/jmscr/v6i10.104

Journal Of Medical Science And Clinical Research

\title{
Quality of life of university students with acne
}

\author{
Authors \\ Inder Pal Singh, $\mathrm{MD}^{1}$, Rajwinder Singh, $\mathrm{MD}^{2}$, Vinay Shanker, $\mathrm{MD}^{3}$ \\ Simran Singh Aujla, $\mathrm{MD}^{4}$ \\ ${ }^{1}$ Assistant Professor, ${ }^{2}$ Associate Professor, ${ }^{3}$ Professor and Head, ${ }^{4}$ Senior Resident
}

Department of Dermatology, Venereology and Leprosy, Maharishi Markandeshwar Medical College and

Hospital, Kumarhatti, Solan - 173229, Himachal Pradesh, India

Corresponding Author

Rajwinder Singh, MD

Associate Professor, Department of Dermatology, Venereology and Leprosy, Maharishi Markandeshwar Medical College and Hospital, Kumarhatti, Solan - 173229, Himachal Pradesh, India

Phone 09501300469, Email: drrajgomco@gmail.com

\begin{abstract}
Background: The psychological consequences of having acne have often been underestimated. Assessment of the acne related quality of life can give us an accurate insight into how patients live with their acne. According to WHO, health care providers must incorporate quality of life issues in patient assessment. This study aims to assess the quality of life impairment due to acne among university students with acne.

Materials and Methods: 900 university students were administered the acne specific quality of life questionnaire - the Cardiff acne disability index (CADI). A targeted history and examination was completed for those who reported acne. Age, gender and severity of acne were correlated with CADI scores.

Results and Conclusion: Acne impacted the quality of life of university students with acne. Our study showed a significant association between severity of acne and quality of life impairment. Female students experienced a greater negative impact of acne. Physicians must consider the psychosocial burden of acne in addition to pharmacologic treatment. We recommend that quality of life issues must be evaluated in all patients with acne for patient centred comprehensive management.

Keywords: Health related quality of life (HRQoL), Quality of life impairment (QUIMP), acne, Cardiff acne disability index (CADI), Himachal Pradesh (India).
\end{abstract}

\section{Introduction}

Most people experience acne during adolescence. More than $95 \%$ of teenage boys and $85 \%$ of teenage girls experience acne. $50 \%$ of these adolescents continue to suffer from acne in adulthood. ${ }^{1}$ Acne is a chronic disease and it causes profound negative psychological and social effects on the quality of life (QoL) of patients ${ }^{2}$. Previous studies have documented problems with perception of self, and social and emotional problems in acne patients ${ }^{1,3}$. To assess these problems, measurement of health related quality of life (HRQoL) is recommended as an integral part of comprehensive acne management. To 
measure the HRQoL in acne, a valid and reliable acne specific instrument such as Cardiff Acne Disability Index (CADI) is used. ${ }^{4}$ HRQoL impairment of acne patients has been reported in studies from the United States ${ }^{5}$, Greece ${ }^{6}$, Oman $^{7}$, Saudi Arabia ${ }^{8}$, Malaysia ${ }^{9}$, Ukraine ${ }^{10}$, Serbia ${ }^{11}$ and $\operatorname{Iran}^{12}$. The new word "QUIMP" meaning "quality of life impairment" was recently proposed by Professor Finlay $^{13}$; and recommended by the "European Academy of Dermatology and Venereology Task Forces on Quality of Life and Patient Oriented Outcomes" for routine clinical and research use. ${ }^{14}$ In India, the effect of acne on HRQoL is undocumented in this part of the country. The aim of this study is to evaluate the HRQoL of university students suffering from acne using the Cardiff acne disability index (CADI). The study analyzes the impact of acne severity, age, and gender on quality of life impairment (QUIMP) among university students.

\section{Materials and Methods}

The study was a cross sectional observational survey done over a period of first two weeks of September 2018. 900 students of Maharishi Markandeshwar University at Solan in Himachal Pradesh, India, were requested to fill in acne specific HRQoL questionnaire - the CADI and a short sociodemographic questionnaire including age, gender, duration of acne, family history of acne, co morbid health conditions and medications. The questionnaires were administered by a dermatologist in each class room. The participation was anonymous and on a voluntary basis; and students were free to refuse to complete the questionnaire if they wished. The study was approved by the institutional ethics committee. Students who self reported acne were objectively evaluated by the dermatologist doing a targeted history and examination. The morphology and site of acne lesions was assessed and the acne was graded as follows:

- Grade 1 - comedones and occasional papules

- Grade 2 - papules, comedones and a few pustules
- Grade 3 - predominant pustules, nodules and abscesses

- Grade 4 - mainly cysts, abscesses, and widespread scarring

The Cardiff Acne Disability Index (CADI) is a well validated, short 5 item questionnaire with a Likert scale designed for use in teenagers and young adults with acne $e^{4}$. It is self explanatory and is usually completed in one minute. Permission for use of the CADI questionnaire was obtained from Professor A Y Finlay. A printed copy of the questionnaire was simply handed over to each student in the class to fill in and return. The 5 questions or domains explored in the CADI are:

1. Have you been aggressive, frustrated or embarrassed as a result of your acne?

2. Do you think that your social life or relationships were affected as a result of your acne?

3. Have you avoided public changing facilities or swimming as a result of your acne?

4. How would you describe your feelings about the appearance of your skin?

5. How bad you think your acne is now?

Items 1 through 4 relate to the patient's feelings, emotions and areas of concern because of acne over the last month. Item 5 is an indication of the self reported perceived severity of acne at present. Each item or question has 4 response options or choices and is scored from 0 to 3 . The CADI score is calculated by summing the score of each question resulting in a possible maximum of 15 and a minimum of 0 . Total scores were classified as low (0-4), medium (5-9), and high (10-15). The higher the score, the more the quality of life is impaired (QUIMP).

Only those students who reported active acne were included in the data analysis. Students with a pre-existing psychiatric diagnosis and other medical and skin disorders which affect HRQoL were excluded as were those on medications likely to interfere with assessment of acne.

Data were analyzed with chi square test used to assess significant difference between groups. The 
level of significance was set at $\mathrm{p}<0.05$. The categorical values were summarized as proportions and percentages. The continuous variables were summarized as means.

\section{Results}

Overall, 753 (84\%) out of 900 university students surveyed reported acne. Out of 753 students with acne, $510(68 \%)$ were females and 243 (32\%) were males. The female to male ratio was approximately 2:1. Age of students surveyed ranged from 17 to 26 years with a mean of 21 years. The highest prevalence of acne was at age 17-20 (Table 1). The most commonly observed lesion was a comedone (Figure 1). The most common location of acne lesions was the face (94\%) followed by face and trunk $(6 \%)$. Grade 1 acne was the most common followed by grade 2 acne (Table 2). We also found that a higher proportion of males ( 85 out of 243 or $34.9 \%$ ) than females (34 out of 510 or $6.6 \%$ ) suffered from grade 3 and 4 acne (Table 2). No significant association ( $\mathrm{p}$ value 0.15 ) was observed between severity of acne and age (Table 3 ). The overall mean CADI score was low (3.7). Majority (81\%) of the patients had low (0-4) CADI scores (Table 4). CADI scores were also low in majority of the students in all three age groups (Table 5). We observed that as the grade of acne increased, there was significant quality of life impairment (QUIMP) indicated by higher CADI scores ( $\mathrm{p}<$ 0.05 ). A significant number ( $p$ value $<0.5$ ) of female students had medium to high CADI scores as compared to male students (Table 6). Low (04), medium (5-9) and high (10-15) CADI values indicate low, medium and high degree of quality of life impairment respectively.

Table1. Age and gender distribution of students

\begin{tabular}{|l|c|c|c|}
\hline Age & Male & Female & Total $(\%)$ \\
\hline $17-20$ & 101 & 297 & $398(52.8)$ \\
\hline $21-24$ & 107 & 188 & $295(39.2)$ \\
\hline$>24$ & 35 & 25 & $60(8)$ \\
\hline All $(\%)$ & $243(32)$ & $510(68)$ & $753(100)$ \\
\hline
\end{tabular}

Table 2 Acne grades and gender distribution

\begin{tabular}{|l|c|c|c|}
\hline Grade & Male & Female & Total (\%) \\
\hline Grade 1 & 36 & 286 & $322(42.8)$ \\
\hline Grade 2 & 122 & 190 & $312(41.4)$ \\
\hline Grade 3 & 82 & 34 & $116(15.4)$ \\
\hline Grade 4 & 3 & 0 & $3(0.4)$ \\
\hline All grades (\%) & $243(32)$ & $510(68)$ & $753(100)$ \\
\hline
\end{tabular}

Prevalence of grade 3 and 4 acne in males $(85 \div 243$ or $34.9 \%)$; in females $(34 \div 510$ or $6.6 \%)$; overall prevalence of grade 3 and 4 acne $119 \div 753(15.8 \%)$

Figure1. Percentages of students with different grades of acne

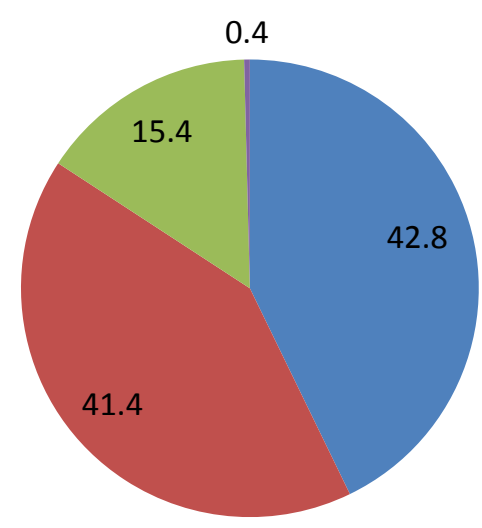

$$
\begin{aligned}
& \text { Grade1 (comedones) } \\
& \text { Grade } 2 \text { (papules and } \\
& \text { comedones) } \\
& \text { Grade } 3 \text { (pustules, nodules) } \\
& \text { Grade } 4 \text { (cysts, abscesses, } \\
& \text { scars) }
\end{aligned}
$$


Table 3 Acne grades and age of students

\begin{tabular}{|l|c|c|c|c|}
\hline Grade & Age 17-20 & Age 21-24 & Age >24 & Total $(\%)$ \\
\hline Grade 1 & 178 & 121 & 23 & $322(42.8)$ \\
\hline Grade 2 & 169 & 118 & 25 & $312(41.4)$ \\
\hline Grade 3 & 51 & 54 & 11 & $116(15.4)$ \\
\hline Grade 4 & 0 & 2 & 1 & $3(0.4)$ \\
\hline All grades (\%) & $398(52.8)$ & $295(39.2)$ & $60(8)$ & $753(100)$ \\
\hline
\end{tabular}

Table 4 Acne grades and numbers of students with low, medium and high CADI scores*

\begin{tabular}{|l|c|c|c|c|}
\hline Grade & CADI 0-4 & CADI 5-9 & CADI 10-15 & Total (\%) \\
\hline Grade 1 & 300 & 12 & 10 & $322(42.8)$ \\
\hline Grade 2 & 270 & 35 & 7 & $312(41.4)$ \\
\hline Grade 3 & 40 & 65 & 11 & $116(15.4)$ \\
\hline Grade 4 & 0 & 0 & 3 & $3(0.4)$ \\
\hline All grades (\%) & $610(81)$ & $112(15)$ & $31(4)$ & $753(100)$ \\
\hline
\end{tabular}

*Low CADI 0-4, medium CADI 5-9, high CADI 10-15

Number of students with CADI score 5-15 is $143 \div 753(19 \%)$

Table 5 Age and number of students with low, medium and high CADI scores*

\begin{tabular}{|l|c|c|c|c|}
\hline Age & CADI 0-4 & CADI 5-9 & CADI 10-15 & Total (\%) \\
\hline $17-20$ & 300 & 80 & 18 & $398(52.8)$ \\
\hline $21-24$ & 260 & 30 & 5 & $295(39.2)$ \\
\hline$>24$ & 50 & 2 & 8 & $60(8)$ \\
\hline All $(\%)$ & $610(81)$ & $112(15)$ & $31(4)$ & $753(100)$ \\
\hline
\end{tabular}

*Low CADI 0-4, medium CADI 5-9, high CADI 10-15

Table 6 Gender and number of students with low, medium and high CADI scores*

\begin{tabular}{|l|c|c|c|c|}
\hline Gender & CADI 0-4 & CADI 5-9 & CADI 10-15 & Total (\%) \\
\hline Male & 200 & 40 & 3 & $243(32.3)$ \\
\hline Female & 410 & 72 & 28 & $510(67.7)$ \\
\hline Both $(\%)$ & $610(81)$ & $112(15)$ & $31(4)$ & $753(100)$ \\
\hline
\end{tabular}

*Low CADI 0-4, medium CADI 5-9, high CADI 10-15

\section{Discussion}

753 out of 900 university students reported acne. Therefore, the prevalence of self reported acne in our study is $83.66 \%$. The results of our study revealed that majority of the students $(52.8 \%)$ were 17-20 years of age. Therefore, this study confirms that acne is common in teenagers. This is in concordance with many other studies. ${ }^{6}, 15,16$ However; the comparison of different studies is, in general, difficult because of differences in study designs, settings and population characteristics. Out of 753 students who reported acne, $510(67.7 \%)$ were females. Therefore, a higher proportion of females than males reported acne. This is confirmed by other studies ${ }^{16,17}$. Acne is more common in females due to hormonal factors and use of cosmetics. However, this may be simply because, in our culture, females are more conscious of their appearance than males and report their acne more frequently. In a study from Serbia, a higher proportion $(64.3 \%)$ of males reported acne. ${ }^{11}$ In our study, the most common site of acne was face (94\%). In a study from South India, $99.3 \%$ of the patients had facial acne ${ }^{18}$. Prevalence of facial acne was $89 \%$ in a study from Greece $^{6}$. Since facial acne affects the appearance of the individual, it produces a disturbance of body image and may be a concern for the patient. The most common types of acne lesions in our study were come dones and papules (84\%). This figure was $65.82 \%$ in a study from Greece ${ }^{6}$, and $95 \%$ in a study from South India ${ }^{18}$. In our study $143(19 \%)$ out of 753 students had medium to high QUIMP (CADI scores 5-15) as seen in Table 4. Therefore, although acne is often viewed as a minor cosmetic issue, it can have a negative impact on psychosocial functioning. However, patients in a study from Sarawak in Malaysia were 
least affected in the domain of psychosocial functioning, which may be attributed to the cultural attitudes and upbringing of this population encouraging stoicism ${ }^{9}$. In our study, the QUIMP or psychosocial impairment according to CADI scoring showed a positive correlation with acne severity. This means that the QUIMP became worse as the severity of acne increased. However, 17 (2.7\%) out of 634 patients with grade 1 and 2 acne had high CADI scores ranging from 10 to 15 (and therefore, high QUIMP) as seen in Table 4. Thus, the HRQoL of an individual does not always correlate with the physician's assessment of acne severity, underlining the need for effective and timely management of acne even for patients with less severe acne. This point must be stressed in the training of physicians and medical students, as the treatment of acne should not be based on the severity of acne alone but also on the degree of QUIMP. Overall, the prevalence of grade 3 and 4 acne in our study was $15.8 \%$. However, it was much higher in males $(34.9 \%)$ as compared to females $(6.6 \%)$. The higher prevalence of more severe acne among males has been observed in other studies. ${ }^{17,19}$ However, in our study, female students experienced a greater QUIMP because of their acne. This means that girls experienced more psychological morbidity due to acne than boys. In a population study of 1531 school pupils from Greece, the HRQoL of girls and boys were equally affected in those with acne ${ }^{8}$. A study from Egypt $^{20}$ reported greater impairment of the quality of life among male adolescents with acne while a study from China $^{21}$ reported greater impairment of life among female adolescents with acne. In a study from South India, both males and females were concerned about their acne ${ }^{18}$ Acne, especially when severe, may have an impact on the life of patients comparable to that caused by other chronic diseases such as asthma, epilepsy, diabetes, back pain and arthritis ${ }^{22}$. In the present study, we detected QUIMP or psychosocial impairment caused by acne among all the age groups of university students and in both genders.
We observed that female students experienced a greater overall negative impact on their quality of life. This suggests that adolescent and young adult females may be more susceptible than boys to the negative psychological effects of acne.

Our study is limited in that it is a questionnaire based cross sectional study of university students and our results probably cannot be applied to the general population. The questionnaire helped us to assess psychosocial functioning but was insufficient to diagnose psychiatric co morbidity. The strengths of this study include a large sample size and use of a specific scale (CADI) to assess the HRQoL among acne patients. The clinical evaluation by the dermatologist of self reported acne adds strength to the study.

\section{Conclusion}

Our study showed that acne affects the HRQoL of the affected students. Mild acne does not mean little impact on HRQoL. Many students with mild acne reported that they felt aggressive, frustrated or embarrassed as a result of having acne. Acne severity correlated significantly with impairment of HRQoL. All the age groups studied and both genders experienced the negative impact on their HRQoL . The HRQoL was worse among female students with acne. Therefore, health care providers should assess the psychosocial and emotional aspects while managing patients with acne. This is because effective therapies of acne are available, and administration of these agents can improve the HRQoL of these patients. We recommend adopting a $\mathrm{HRQOL}$ measure as an integral part of comprehensive acne assessment so as to assist the physician in making better clinical decisions.

\section{Acknowledgements}

We thank Professor AY Finlay, Department of Dermatology, University of Wales College of Medicine, Cardiff, Wales, United Kingdom, for the formal permission to use the CADI questionnaire in this study. We also express our gratitude to Ms Sneha, Assistant Professor cum 
Statistician at our institute for help with the statistical analysis.

Sources of support in the form of grants: nil

\section{References}

1. Ghodsi SZ, Orawa H, Zouboulis CC. Prevalence, severity, and severity risk factors of acne in high school pupils: a community-based study. J Invest Dermatol. 2009 Sep;129(9):2136-41.

2. Gollnick HPM, Finlay AY, Shear N, Global Alliance to Improve Outcomes in Acne. Can we define acne as a chronic disease? If so, how and when? Am J Clin Dermatol. 2008;9(5):279-84.

3. Dunn LK, O'Neill JL, Feldman SR. Acne in adolescents: quality of life, self-esteem, mood, and psychological disorders. Dermatol Online J. 2011 Jan 15;17(1):1.

4. Motley RJ, Finlay AY. Practical use of a disability index in the routine management of acne. Clin Exp Dermatol. 1992 Jan;17(1):1-3.

5. Gorelick J, Daniels SR, Kawata AK, Degboe A, Wilcox TK, Burk CT, et al. Acne-Related Quality of Life Among Female Adults of Different Races/ Ethnicities. J Dermatol Nurses Assoc. 2015 May;7(3):154-62.

6. Tasoula E, Gregoriou S, Chalikias J, Lazarou D, Danopoulou I, Katsambas A, et al. The impact of acne vulgaris on quality of life and psychic health in young adolescents in Greece. Results of a population survey. An Bras Dermatol. 2012 Dec;87(6):862-9.

7. Al-Shidhani A, Al-Rashdi S, Al-Habsi H, Rizvi S. Impact of Acne on Quality of Life of Students at Sultan Qaboos University. Oman Med J. 2015 Jan;30(1):42-7.

8. Kokandi A. Evaluation of Acne Quality of Life and Clinical Severity in Acne Female Adults [Internet]. Dermatology Research and Practice. 2010 [cited 2018 Sep 26].
Available

from:

https://www.hindawi.com/journals/drp/201 0/410809/

9. Yap FB-B. The impact of acne vulgaris on the quality of life in Sarawak, Malaysia. J Saudi Soc Dermatol Dermatol Surg. 2012 Jul 1;16(2):57-60.

10. Pochynok T, P Chernyshov I, Asayevich N, Sushko S, Kopylova V, V Chernyshov P. Quality of life of school and university students with acne. Acta Dermatovenerol Croat. 2018 Jul 5;26(2):139-139.

11. Jankovic S, Vukicevic J, Djordjevic S, Jankovic J, Marinkovic J. Quality of life among schoolchildren with acne: Results of a cross-sectional study. Indian $\mathbf{J}$ Dermatol Venereol Leprol. 2012 Jul 1;78(4):454.

12. Ghaderi R, Saadatjoo A, Ghaderi F. Evaluating of life quality in patients with acne vulgaris using generic and specific questionnaires. Dermatol Res Pract. 2013;2013:108624.

13. Finlay AY. Quimp: A Word Meaning "Quality of Life Impairment." Acta Derm Venereol. 2017 06;97(4):546-7.

14. Chernyshov PV, Zouboulis CC, TomasAragones L, Jemec GB, Manolache L, Tzellos T, et al. Quality of life measurement in acne. Position Paper of the European Academy of Dermatology and Venereology Task Forces on Quality of Life and Patient Oriented Outcomes and Acne, Rosacea and Hidradenitis Suppurativa. J Eur Acad Dermatol Venereol JEADV. 2018 Feb;32(2):194208.

15. Kubota Y, Shirahige Y, Nakai K, Katsuura J, Moriue T, Yoneda K. Community-based epidemiological study of psychosocial effects of acne in Japanese adolescents. J Dermatol. 2010 Jul;37(7):617-22.

16. Yeung CK, Teo LHY, Xiang LH, Chan HHL. A community-based epidemiological study of acne vulgaris in 
Hong Kong adolescents. Acta Derm Venereol. 2002;82(2):104-7.

17. Hanisah A, Omar K, Shah SA. Prevalence of acne and its impact on the quality of life in school-aged adolescents in Malaysia. $\mathbf{J}$ Prim Health Care. 2009 Mar;1(1):20-5.

18. Durai PCT, Nair DG. Acne vulgaris and quality of life among young adults in South India. Indian J Dermatol. 2015 Feb;60(1):33-40.

19. Smithard A, Glazebrook C, Williams HC. Acne prevalence, knowledge about acne and psychological morbidity in midadolescence: a community-based study. $\mathrm{Br}$ J Dermatol. 2001 Aug;145(2):274-9.

20. Abdel-Hafez K, Mahran AM, Hofny ERM, Mohammed KA, Darweesh AM, Aal AA. The impact of acne vulgaris on the quality of life and psychologic status in patients from upper Egypt. Int J Dermatol. 2009 Mar;48(3):280-5.

21. Law MPM, Chuh AAT, Lee A, Molinari N. Acne prevalence and beyond: acne disability and its predictive factors among Chinese late adolescents in Hong Kong. Clin Exp Dermatol. 2010 Jan;35(1):16-21.

22. Mallon E, Newton JN, Klassen A, StewartBrown SL, Ryan TJ, Finlay AY. The quality of life in acne: a comparison with general medical conditions using generic questionnaires. $\mathrm{Br} \quad \mathrm{J}$ Dermatol. 1999 Apr;140(4):672-6. 